\title{
Congenital protein hypoglycosylation diseases
}

\author{
This article was published in the following Dove Press journal: \\ The Application of Clinical Genetics \\ 4 July 2012 \\ Number of times this article has been viewed
}

\section{Susan E Sparks \\ Department of Pediatrics, Levine Children's Hospital at Carolinas Medical Center, Charlotte, NC, USA; Department of Pediatrics, University of North Carolina School of Medicine, Chapel Hill, NC, USA}

Correspondence: Susan E Sparks

Carolinas Medical Center, Department

of Pediatrics/Clinical Genetics,

CIR/I st Floor, I I00 Blythe Boulevard,

Charlotte, NC 28203, USA

$\mathrm{Tel}+\mathrm{I} 70438 \mathrm{I} 6810$

Fax + I 704 38I 68II

Email susan.sparks@carolinashealthcare.org
Abstract: Glycosylation is an essential process by which sugars are attached to proteins and lipids. Complete lack of glycosylation is not compatible with life. Because of the widespread function of glycosylation, inherited disorders of glycosylation are multisystemic. Since the identification of the first defect on N-linked glycosylation in the 1980s, there are over 40 different congenital protein hypoglycosylation diseases. This review will include defects of $\mathrm{N}$-linked glycosylation, O-linked glycosylation and disorders of combined N- and O-linked glycosylation.

Keywords: congenital disorders of glycosylation, dystroglycanopathies, hypoglycosylation, glycoproteins

\section{Introduction}

Glycosylation of proteins is a post-translational process that is important for many proteins to function. The addition of carbohydrates to the proteins stabilizes the protein, is involved in cell-cell interaction and cellular signaling, and modulates protein function. Defects in glycosylation encompass abnormalities in the synthesis of N-linked glycoproteins, O-linked glycoproteins, or both, as well as a defect in lipid-glycosylation. Since the characterization of N-linked defects in the 1980s, the field of clinical glycobiology has rapidly progressed and now includes defects in glycosyltransferases for some forms of muscular dystrophy.

Protein glycosylation is defined as the synthesis of glycans and their covalent attachment to proteins. Glycans are also attached to lipids forming glycolipids (glycophospholipids, glycosphingolipids). Approximately $0.5 \%-1 \%$ of the transcribed human genome is responsible for the synthesis, degradation, and function of glycoconjugates. ${ }^{1}$ Since about half of the body proteins are glycoproteins, this is an immense field of study.

Glycoproteins are classified by the type of linkage of the oligosaccharides to the peptide. This attachment can be either N-linked through the amide group of selected asparagine residues to an $N$-acetylglucosamine (GlcNAc) residue, or O-linked through the hydroxyl group mainly of serine or threonine residues via $N$-acetylgalactosamine (GalNAc), mannose (Man), xylose, or other monosaccharide residues.

In general, the process of protein glycosylation occurs in fours stages that take place in the cytosol, the endoplasmic reticulum (ER), and the Golgi compartments. In the first stage, monosaccarides are activated in the cytosol (Figure 1). These monosaccharides are derided from dietary sources as well as salvage pathways in the cell. 


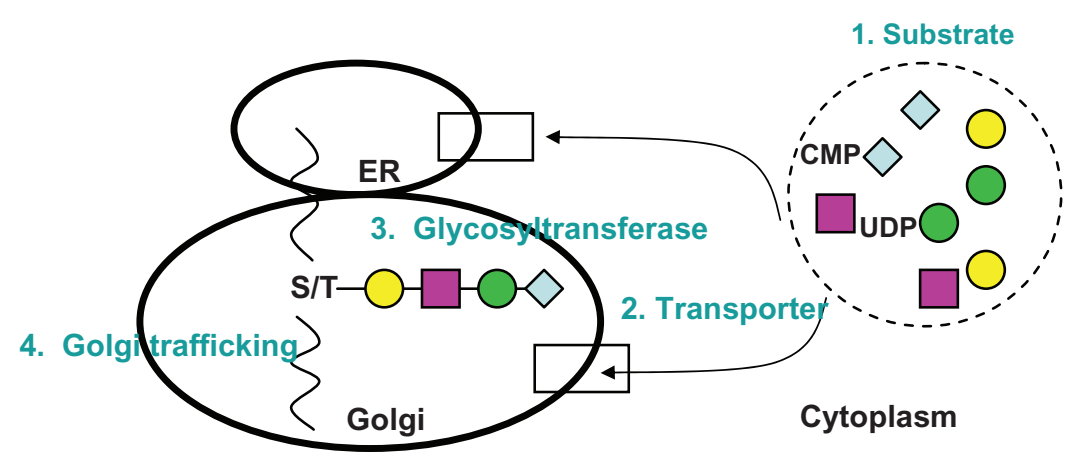

Figure I Overview of glycosylation biosynthesis pathway.

Abbreviations: ER, endoplasmic reticulum; CMP, cytosine monophosphate; S/T, serine/threonine; UDP, uridine diphosphate.

Activated monosaccharides are generated through a series of phosphorylation, epimerization, and acetylation reactions. These become the high-energy nucleotide sugar donors.

The next stage involves transport of the sugar donors from the cytoplasm into the lumen of the ER, by binding to dolichol phosphate, and the Golgi by specific transporters. For transport into the ER, the nucleotide sugars bind to the cytosolic side of the dolichol phosphate which is membrane bound. A "flippase" mediates the transfer of the sugar into the luminal side of the ER. Defects in either the synthesis of the dolichol phosphate [DPM1-CDG (CDG-Ie)], or in the transfer LEC35-CDG (CDG-If) result in clinical disease (see below). In the Golgi, there are specific nucleotide sugar transporters to transfer the activated monosaccharides to the luminal side. Abnormal glycosylation can be seen if these transporters are deficient, such as the GDP-Fucose transporter in SLC35C1-CDG (CDG-IIC).

In the third stage, specific glycosyltransferases attach the monosaccharides to the growing oligosaccharide. This occurs in a sequential and highly controlled fashion. Most of the $\mathrm{N}$ - and O-linked defects described to date are deficiencies of a specific glycosyltransferase.

The final stage represents Golgi trafficking and integrity mechanisms. Glycoproteins and glycosyltransferases are transported to their final destination via the cisternae and Golgi vesicles. Defects in the subunits of the conserved oligomeric Golgi (COG) complex result in defective glycosylation.

The biosynthesis of N-linked glycans occurs cotranslationally and is spread over the cytosol, the ER, and the Golgi compartments. In the cytosol and the ER, monosaccharides are attached in a stepwise fashion to form a lipid-linked oligosaccharide (LLO). The monosaccharide donors are either nucleotide- or dolichol phosphate-linked sugars synthesized in the cytosol. These are the substrates for specific glycosyltransferases, which sequentially form the progressively growing LLO in the ER. In the ER, the completed basic glycan $\left(\mathrm{Glc}_{3} \mathrm{Man}_{9} \mathrm{GlNAc}_{2}\right.$, where Glc is glucose, Man is mannose, GlcNAc is N-acetylglucosamine) is transferred en bloc to the asparagine, which is part of the consensus sequence on the protein, Asn-X-Ser/Thr (where $\mathrm{X}$ is any amino acid). This transfer is catalyzed by the oligosaccharyltransferase complex. The glycosylated protein is further processed in the ER and the Golgi into a more complex structure by removal of glucose and mannose residues and the addition of GlcNAc, galactose, fucose, and sialic acid residues. ${ }^{2}$

In contrast, the biosynthesis of O-linked glycans occurs post-translationally and mainly within the Golgi compartment. In addition, there is no consensus sequence to determine the O-glycosylation sites within a protein. However, since O-linked glycosylation occurs after protein folding; only exposed serine and threonine residues are available for glycosylation. There are seven different types of O-glycan attachments in humans, classified by the first sugar (GalNAc, Xyl, GlcNAc, Gal, Man, Glc, Fuc) bound to the protein. The mucin-type O-glycoprotein, which begins with the addition of a GalNAc to a serine or threonine of a fully formed protein, and the glycosaminoglycans (GAGs) where a Xyl is the first sugar, are the most common O-linked glycoproteins. Following this initial step, there is elongation and modification of the oligosaccharides. ${ }^{2}$ Mucin-type O-glycans can be further subdivided into eight core structures depending on the second sugar(s) and/or binding position resulting in at least 50 different types. The GAGs also occur in various different forms and contain 100 or more monosaccharides in their chain. The structures of the other $5 \mathrm{O}$-glycan types show less variability and mostly occur in one conformation. ${ }^{3}$

A specific type of O-glycosylation involves the attachment of a mannose. While many brain glycoproteins contain O-linked mannose oligosaccharides, only $\alpha$-dystroglycan has been well characterized. ${ }^{4}$ Alpha-dystroglycan is part of the dystrophin-glycoprotein complex which is important for 
membrane stability and cell signaling functions in the central nervous system as well as muscle. Alpha-dystroglycan is heavily glycosylated with N-linked oligosaccharides and mucin-type O-linked oligosaccharides. In addition, it has O-linked mannose oligosaccharides. This glycan type is initiated in the ER by the combined action of protein-Omannosyltransferases, POMT1/POMT2 and uses dolicholphosphate activated mannose as a substrate. The glycan is further elongated by a specific GlcNAc-transferase (encoded by $P O M G n T 1$ ) in the Golgi. Additional glycosyltransferases yet to be identified extend the glycan with a galactose and a sialic acid residue. Defects in POMT1/POMT2 and $P O M G n T 1$ cause abnormal glycosylation of $\alpha$-dystroglycan and result in muscular dystrophy.

\section{Clinical diagnoses}

Abnormalities in the synthesis of N-linked glycoproteins, O-linked glycoproteins or both will be reviewed. By far the most abundant and well studied of the protein hypoglycosylation defects are the group of disorders known as Congenital Disorders of Glycosylation (formerly known as carbohydrate-deficient glycoprotein syndrome-CDGS) which are defects in the elongation pathway of the biosynthesis of $\mathrm{N}$-glycans that occurs in the ER (type I CDG) and defects in the processing pathway in the ER and Golgi (type II CDG). Due to the utilization of some of the enzymes in the biosynthesis of $\mathrm{N}$-glycans and O-glycans, several of the subtypes of CDG affect O-glycosylation as well.

In the O-linked glycoprotein pathway, clinically deleterious defects in three particular branches have been elucidated. First, a defect in O-GalNAc glycans is responsible for familial tumoral calcinosis. Second, defects in O-mannosylation are responsible for the dystroglycanopathies which cause several types of muscular dystrophy. Hereditary inclusion body myopathy (HIBM) is caused by a defect in sialic acid biosynthesis and has also been shown to have a defect in $\mathrm{O}$-mannosylation in muscle, and is discussed with this group as well. Third, O-xylosylglycan defects are responsible for a progeroid variant of Ehlers-Danlos syndrome and hereditary multiple exostoses syndrome.

CDG was initially described in identical twin sisters with developmental delay, decreased thyroxine-binding globulin levels and increased lysosomal enzyme activities. Further analysis using isoelectric focusing (IEF) of serum transferring demonstrated a cathodal shift of their transferring IEF profile, which is now considered pathognomonic for the diagnosis of CDG. ${ }^{2,5}$ With the exception of MPI-CDG (CDG-Ib), which primarily presents with protein-losing enteropathy, the other subtypes of N-linked glycosylation defects all present with a combination of growth and developmental delay, hypotonia, and variable involvement of multiple organ systems. Initially the nomenclature for this group of disorders was CDG followed by the type of transferrin isoelectric focusing pattern (I or II) and the letter in order of the identified gene defect (ie, CDG-Ia or CDG-IIa). With the identification of many more types of glycosylation defects, this nomenclature was changed with the gene name, followed by "CDG", and the older nomenclature in parenthesis (ie, PMM2-CDG $(C D G-I a)){ }^{6}$

\section{N-linked glycosylation defects}

- PMM2-CDG (CDG-Ia). This is the most common, with over 700 patients described worldwide, and the prototype for the group of the CDGS. It is a multisystem disorder characterized by inverted nipples, abnormal subcutaneous fat distribution, and cerebellar hypoplasia, in combination with hypotonia and developmental delay. In infancy, there is failure to thrive with feeding difficulties and gastroesophageal reflux. Serum liver transaminases are elevated and hypoalbuminemia can occur leading to edema and even anasarca. Clotting factors, particularly Factor XI, antithrombin III, and protein C, can be decreased leading to bleeding following minor trauma or surgery. Seizures are common and there can be stroke-like episodes. Pericardial effusions, renal cysts, retinitis pigmentosa, multiple infections and endocrine abnormalities have been described. The frequency of mortality in infancy is $20 \%-25 \%{ }^{2,7}$ Adults with PMM2-CDG (CDG-Ia) have developmental delay, ataxia, peripheral neuropathy, scoliosis, and hypergonadotropic hypogonadism. ${ }^{8}$ Recent reports have widened the phenotypic spectrum to include hydrops fetalis at the severe end ${ }^{9}$ and a mild neurologic phenotype in adults with multisystemic involvement at the mild end. ${ }^{10,11}$ Reports indicate that the prevalence may be as high as $1: 20,000^{5}$ and the carrier frequency in the Danish population may be as high as $1: 60-1: 79 .{ }^{12}$ It is likely that this disorder is under recognized.

- MPI-CDG (CDG-Ib). Cyclic vomiting, profound hypoglycemia, failure to thrive, liver fibrosis, and protein-losing enteropathy, occasionally associated with coagulation disturbances without neurologic involvement, are characteristic. The clinical course is variable even within families. Taken orally, the sugar mannose appears to improve the growth and gastrointestinal manifestations of this disorder. ${ }^{13-15}$ At least 20 individuals with this diagnosis have been described. 
- ALG6-CDG (CDG-Ic). ALG6-CDG (CDG-Ic) was previously classified as carbohydrate-deficient syndrome type $\mathrm{V}^{16}$ and is characterized by mild-to-moderate neurologic involvement with hypotonia, poor head control, developmental delay, ataxia, strabismus, and seizures, ranging from febrile convulsions to epilepsy. ${ }^{17-19}$ The clinical presentation may be milder than in PMM2-CDG $(C D G-I a)$ and stroke-like episodes and peripheral neuropathy have not been reported. However, retinal degeneration has been reported. ${ }^{19}$ An adult with ALG6-CDG $(C D G-I c)$ was described with brachydactyly, deep vein thrombosis, pseudotumor cerebri with normal brain MRI, and endocrine abnormalities including hyperandrogenism with virilization..$^{20}$ Pubertal abnormalities have been described in an individual with ALG6-CDG (CDG-Ic). ${ }^{21}$ Over 30 patients with ALG6-CDG (CDG-Ic) have been described.

- ALG3-CDG (CDG-Id). There have been five children described with defects in ALG3. They all had severe psychomotor delay, microcephaly, and severe seizures. Vision impairment was described in 3 infants, 2 with optic atrophy and iris coloboma. Arthrogryposis was described in one of the children. Siblings were described where one had significant digestive issues while the other was more neurologically impaired. ${ }^{22-25}$

- DPM1-CDG (CDG-Ie). Five individuals had severe developmental delay, microcephaly, seizures, ocular hypertelorism, a "gothic palate," small hands with dysplastic nails, and knee contractures. ${ }^{26-29}$ Additionally, two sibs were described with a milder phenotype, consisting of developmental delay, microcephaly, ataxia, and peripheral neuropathy without distinctive features or severe seizures. ${ }^{30}$ They had nystagmus and strabismus; one had a retinopathy.

- MPDU1-CDG (CDG-If). Five individuals had severe psychomotor retardation, generalized scaly, erythematous skin, and attacks of hypertonia. In addition, short stature, seizures, and vision impairment have been described. ${ }^{31,32}$

- ALG12-CDG (CDG-Ig). Seven individuals had distinctive features, generalized hypotonia, feeding difficulties, moderate to severe psychomotor retardation, progressive microcephaly, frequent upper respiratory tract infections, impaired immunity with decreased immunoglobulin levels, and decreased coagulation factors. ${ }^{33-39}$ Additional features included hypogonadism with or without hypospadias in the males, seizures in two individuals, and cardiac anomalies in two sibs.
- ALG8-CDG (CDG-Ih). A four-month-old female had moderate hepatomegaly, severe diarrhea, and hypoalbuminemia from protein-losing enteropathy, normal facial features, and normal development, similar to PMI-CDG $(C D G-I b) \cdot{ }^{40}$ She had decreased levels of factor XI, protein $\mathrm{C}$, and antithrombin III. Three other affected individuals had cardiorespiratory difficulties with lung hypoplasia, a severe hepatointestinal disorder, and hypotonia. ${ }^{41}$ Two additional patients were described with hypotonia, edema, seizures and psychomotor delay. ${ }^{42,43}$ All five individuals had hematopoietic issues with anemia and thrombocytopenia, and early death between ages three days and 16 months. More recently, two siblings with milder disease were described with hypotonia, cognitive impairment, pseudogynecomastia, and ataxia. ${ }^{44}$

- ALG2-CDG (CDG-Ii). A six-year-old had bilateral iris colobomas, unilateral cataract, infantile spasms beginning at age four months, and severe developmental delay; coagulation factors were abnormal. ${ }^{45}$

- DPAGT1-CDG (CDG-Ij). The one affected individual described had hypotonia, intractable seizures, developmental delay, and microcephaly. ${ }^{46}$

- ALG1-CDG (CDG-Ik). Four affected individuals had severe developmental delay, hypotonia, and earlyonset seizures; the latter were intractable in three. Three individuals died between ages two weeks and ten months. As in ALG3-CDG (CDG-Id) and ALG12CDG (CDG-Ig), also caused by mannosyltransferase defects, microcephaly was rapidly progressive. Other features included severe coagulation defects, nephrotic syndrome, liver dysfunction, coagulation abnormalities, cardiomyopathy, and immunodeficiency. ${ }^{47-49}$ Brain imaging showed cerebral atrophy in two individuals and was normal in a third individual. Further studies have shown that ALG1-CDG (CDG-Ik) and PMI-CDG $(C D G-I b)$ may be the most frequent after PMM2-CDG $(C D G-I a)$ and present at the severe end of the CDG I clinical spectrum. ${ }^{50}$

- ALG9-CDG (CDG-IL). Three children had microcephaly, hypotonia, developmental delay, seizures, and hepatomegaly. ${ }^{51-53}$ Two individuals also had failure to thrive and pericardial effusions. Renal cysts and inverted nipples were also described.

- DOLK-CDG (CDG-Im). Four affected infants had hypotonia and ichthyosis, and died between ages four and nine months. ${ }^{54}$ Additional features included seizures and progressive microcephaly in one and dilated cardiomyopathy in two sibs. 
- RFT1-CDG (CDG-In). An infant born preterm to unrelated parents had difficulty feeding as a result of an uncoordinated suck and failure to thrive. Myoclonic jerks were noted at three weeks with hypotonia and brisk reflexes progressing to a seizure disorder. Exam was also notable for roving eye movements with normal ERG and reduced VEP. At age two years the child continues to have marked developmental delay. ${ }^{55-57}$ Five additional affected individuals have been described. ${ }^{58,59}$ The common features in all six children include severe developmental delay, hypotonia, visual disturbances, seizures, feeding difficulties, and sensorineural hearing loss. Also associated are features similar to other types of CDG including inverted nipples and microcephaly.

- ALG11-CDG (CDG-Ip). A single infant presented with distinctive features (microcephaly, high forehead, and low posterior hairline), hypotonia, and failure to thrive. She had severe neurologic impairment with frequent and difficult to treat seizures. She developed an unusual fat pattern around 6 months of age. She had persistent vomiting and stomach bleeding and passed away at 2 years of age. ${ }^{60}$

- MAGT1-CDG. This is a defect in a subunit of the oligosaccharyltransferase complex. Reported in a family with two girls with mild cognitive impairment and two boys with more severe cognitive involvement. The mother is reported to have mild cognitive impairment. ${ }^{61}$

- N33/TUSC3-CDG. Two different kindreds of siblings with non-syndromic intellectual disability were described to have a defect in this subunit of the oligosaccharyltransferase complex. ${ }^{61,62}$

- SRD5A3-CDG. Individuals from seven families were identified with common features including congenital eye malformations (ocular colobomas, optic disc hypoplasia, and variable degree of visual loss), nystagmus, hypotonia, and developmental delay/ intellectual disability. Dermatologic complications or congenital cardiac defects were identified in some individuals. ${ }^{63}$ An additional 12 individuals from nine families were described with cerebellar ataxia and congenital eye malformations. ${ }^{64}$ Additional mutations in SRD5A3 have been identified in people with Kahrizi syndrome, which consists of coloboma, cataract, kyphosis, and intellectual disability. ${ }^{65}$

- MGAT2-CDG (CDG-IIa). Individuals have facial dysmorphism, stereotypic hand movements, seizures, and varying degrees of psychomotor retardation, but no peripheral neuropathy or cerebellar hypoplasia. A bleeding disorder is caused by diminished platelet aggregation. ${ }^{66}$
Scoliosis and respiratory compromise can also occur. $^{67}$

- GCS1-CDG (CDG-IIb) (also known as MOGS-CDG $(\boldsymbol{C D G}-\boldsymbol{I I b}))$. An infant with generalized hypotonia, craniofacial dysmorphism, hypoplastic genitalia, seizures, feeding difficulties, hypoventilation, and generalized edema died at age 2.5 months. ${ }^{68}$

- B4GALT1-CDG (CDG-IId). Mild psychomotor retardation, Dandy-Walker malformation, progressive hydrocephalus, coagulation abnormalities, and elevated serum creatine kinase concentration have been observed. ${ }^{69}$ An additional patient was described with normal development, mild hepatic involvement, and coagulation abnormalities. $^{70}$

- SLC35A1-CDG (CDG-IIf). One affected infant presented at age four months with macrothrombocytopenia, neutropenia, and immunodeficiency, and died at age 37 months of complications from bone-marrow transplantation. ${ }^{71}$

\section{O-glycosylation defects O-mannosylglycan defects also known as the dystroglycanopathies}

Defective glycosylation of $\alpha$-dystroglycan, a component of the dystrophin-glycoprotein (DAG) complex, has been implicated in several subtypes of muscular dystrophy, known as the dystroglycanopathies. The DAG complex is a multicomponent complex linking the intracellular cytoskeleton with the extracellular matrix in muscle. Alpha-dystroglycan also plays a role in neuronal migration, this in addition to muscular dystrophy; patients with severe defective glycosylation of $\alpha$-dystroglycan also demonstrate central nervous system abnormalities. The dystroglycanopathies include the severe congenital muscular dystrophies, Walker-Warburg syndrome (WWS), muscle-eye-brain disease (MEB), and Fukuyama congenital muscular dystrophies (FCMD), as well as nonsyndromic congenital muscular dystrophies, subtype IC and ID, and several subtypes of limb-girdle muscular dystrophy (LGMD), specifically, LGMD2I, LGMD2K, LGMD2M, LGMD2N, and LGMD2O.

WWS is the most severe of the dystroglycanopathies and is typically associated with death of affected individuals by the age of 2 years. The diagnostic hallmark is the combination of severe brain malformations, typically described as the Cobblestone complex, muscular dystrophy, and structural eye abnormalities, particularly microophthalmia and can include cataracts. The Cobblestone complex is a combination of type II lissencephaly, agenesis of the corpus callosum, cerebellar hypoplasia, and enlarged ventricles. ${ }^{72,73}$ 
With a slightly milder phenotype than WWS, MEB is characterized by muscular dystrophy, eye involvement (congenital myopia and glaucoma, retinal hypoplasia without congenital cataracts), developmental delay, and structural brain defects ranging from microcephaly to the Cobblestone

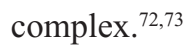

FCMD is seen almost exclusively in Japan. Like WWS and MEB, FCMD is also characterized by muscle and brain involvement, however, only occasionally are eyes involved. ${ }^{72,74}$

Congenital muscular dystrophy type $1 \mathrm{C}$ and type $1 \mathrm{D}$ are caused by defects in the genes FKRP and $L A R G E$ respectively (see below). Like the other congenital muscular dystrophies, there is congenital weakness and developmental delay along with varying degrees of brain malformations, ${ }^{72,75-79}$ and phenotypes as severe as WWS have been described. ${ }^{80,81}$

- POMT1-CDG. POMT1 encodes the protein-Omannosyltransferase 1 that catalyzes the first step in $O$-mannosyl glycan synthesis. ${ }^{82}$ Mutations in POMT1 have been identified in patients with WWS,$^{83}$ a milder congenital muscular dystrophy with calf hypertrophy, microcephaly, and cognitive impairment, ${ }^{84}$ and in patients with LGMD2K..$^{85,86}$

- POMT2-CDG. POMT2 is a second $O$-mannosyltransferase which complexes with POMT1 for the O-mannosyltransferase activity. ${ }^{87,88}$ Mutations in POMT2 have been described in patients with WWS,${ }^{89}$ a MEB-like phenotype, ${ }^{90}$ a milder congenital muscular dystrophy phenotype,${ }^{91}$ and LGMD2N. ${ }^{92}$

- POMGnT1-CDG. Mutations in the POMGnT1 gene which encodes the glycosyltransferase, $O$-mannose $\beta-1,2-N$-acetylglucosaminyltransferase were identified in patients with MEB. ${ }^{93,94}$ Subsequently, mutations in POMGnT1 were identified in patients with $\mathrm{WWS}^{95-98}$ and LGMD2O. ${ }^{99}$ POMGnT1 catalyzes the transfer of $\mathrm{N}$-acetylglucosmine to the $O$-linked mannose of glycoproteins including $\alpha$-dystroglycan.

- FKTN-CDG. The fukutin (FKTN) gene was initially identified to cause FCMD. ${ }^{100-102}$ FKTN mutations have been identified in patients with the more severe phenotype, WWS ${ }^{97,103-105}$ as well as milder non-WWS congenital muscular dystrophy ${ }^{106,107}$ and LGMD2M. ${ }^{108,109}$ Mutations in FKTN have also been identified in patients with isolated hyper-CKaemia ${ }^{110}$ and cardiomyopathy. ${ }^{11,112}$ The fukutin protein shares sequence homology to a bacterial glycosyltransferase, but its precise function is unknown. ${ }^{113} \mathrm{It}$ has been reported that there is colocalization and molecular interaction of fukutin with POMGnT1, suggesting that fukutin may form a complex with POMGnT1 and modulate its enzymatic activity. ${ }^{114}$

- FKRP-CDG. FKRP encodes the fukutin-related protein. Mutations in this gene cause the widest variability in clinical phenotypes, ranging from in utero, severe WWS to very mild LGMD2I. Like fukutin, the function of FKRP is unknown, although it shares homology with FKTN and other glycosyltransferases.

- LARGE-CDG. The LARGE gene is the 5th largest gene in the human genome, spanning $664 \mathrm{~kb}$ of genomic DNA on chromosome 22q12.3-q13.1, and has homology to the glycosyltransferase gene family, ${ }^{115}$ and may have bifunctional activity. ${ }^{116}$ Mutations in LARGE were originally described in MDC1D ${ }^{75,76}$ however, mutations in LARGE have also been described in patients with the severe congenital muscular dystrophy, WWS. ${ }^{80}$

- GNE-CDG. GNE encodes a bifunctional enzyme (UDP-N-acetylglucosamine 2-epimerase/ $\mathrm{N}$-acetlymannosamine kinase) in the biosynthetic pathway of sialic acid, and was identified by linkage analysis to be mutated in hereditary inclusion body myopathy (HIBM). ${ }^{117,118}$ Mutations in GNE cause mostly a distal myopathy, that begins with juvenile onset of muscle weakness which spares the quadriceps and on muscle pathology demonstrates rimmed vacuoles and cytoplasmic and intranuclear inclusions. ${ }^{119,120}$ GNE mutations were also described in another distal myopathy with rimmed vacuoles, Nonaka myopathy. ${ }^{121,122}$

\section{O-xylosylglycan defects}

- 4GalT7-CDG. This defect causes progeroid variant of Ehlers-Danlos syndrome. One patient has been described with premature ageing with loose, hyperelastic skin and joint hyperlaxity. In addition, he had developmental delay, macrocephaly, hypotonia, short stature and osteopenia. $^{2}$

- EXT1/EXT2-CDG. Defects in the Golgi-localized EXT1/EXT2 complex which has both glucuronyltransferase and $N$-acetyl-D-hexosaminyltransferase activities, cause hereditary multiple exostoses syndrome. EXT1/ EXT2-CDG is the most frequent glycosylation defect with an incidence of 1/50,000-100,000 and is the only disorder inherited in an autosomal dominant fashion. It is characterized by benign osteochondromas on the ends of long bones. These lesions are often present at birth, but not usually diagnosed until childhood, progressively grow through adolescence and stop during adulthood. The risk of progression into sarcoma is about $3 \%{ }^{2}$ 


\section{O-fucosylglycan defects}

- B3GALTL-CDG. Also known as Peters Plus syndrome, this disorder is characterized by abnormalities of the anterior chamber of the eye (of which Peters anomaly is the most common), in combination with other systemic symptoms. Included features can be short stature, developmental delay, craniofacial abnormalities including cleft lip with or without cleft palate. ${ }^{23}$

\section{O-GalNAc defects}

- GALNT3-CDG. Defects in the GALNT3 gene cause familial tumoral calcinosis which is a severe disorder involving phosphatemia and massive calcium deposits in the skin and subcutaneous tissue. ${ }^{124}$

\section{Combined N- and O-linked defects}

Many of the glycosylation pathways, specifically the sugar transporters and the Golgi trafficking, are common to both $\mathrm{N}$ - and O-linked glycoprotein synthesis, thus defects in these mechanisms affect both pathways.

- DPM3-CDG (CDG-Io). A single described individual diagnosed with CDG at 27 years had a low normal IQ and mild muscle weakness. She presented initially at age 11 years with mild muscle weakness and waddling gait. She was found to have dilated cardiomyopathy without signs of cardiac muscle hypertrophy at age 20 followed by a stroke-like episode at age $21 .{ }^{125}$ In addition to abnormal $\mathrm{N}$-linked glycosylation, there was abnormal O-linked mannosylation of $\alpha$-dystroglycan (similar to the other dystroglycanopathies).

- SLC35C1-CDG (CDG-IIc). Severe growth and psychomotor retardation, microcephaly, hypotonia, cranofacial dysmorphy, and recurrent bacterial infections with persistent, highly elevated peripheral blood leukocyte count are characteristic. ${ }^{126}$

- COG7-CDG (CDG-IIe). Six affected infants had distinctive features with a small mouth (although one had full lips), microretrognathia, short neck, wrinkled and loose skin, adducted thumbs, and overlapping long fingers; hypotonia; skeletal abnormalities; hepatosplenomegaly; progressive jaundice; seizures; and early death. ${ }^{127-130}$

- COG1-CDG (CDG-IIg). An affected infant presented in the first month of life with feeding difficulties, failure to thrive, and hypotonia. She had mild psychomotor delays, rhizomelic short stature, and progressive microcephaly with slight cerebral and cerebellar atrophy on brain MRI, as well as cardiac abnormalities and hepatosplenomegaly. ${ }^{131}$
- COG8-CDG (CDG-IIh). Two affected infants were reported who had severe psychomotor delay, hypotonia, seizures, esotropia, failure to thrive, and progressive microcephaly. ${ }^{132,133}$

- COG4-CDG (CDG-IIj). The first child described presented at 4 months with complex seizure that was treated with Phenobarbital. At age 3 years, additional findings included hypotonia, microcephaly, ataxia, brisk uncoordinated movements, absent speech, motor delays and recurrent respiratory infections. ${ }^{134}$ A second child presented with failure to thrive, frequent and recurrent infections, diarrhea, hypotonia, hepatosplenomegaly, seizures, elevated liver transaminases, and diffuse cerebral atrophy on brain MRI. ${ }^{135,136}$

- COG5-CDG (CDG-IIk). A single individual was described with mild developmental delay in motor and language. ${ }^{137}$

- COG6-CDG (CDG-IIL). A single infant presented with severe neurologic disease including vitamin K deficiency, intracranial bleeding, vomiting, intractable seizures and early infant death. ${ }^{138}$

- ATP6V0A2-CDG and other cutis laxa. Multiple patients with autosomal recessive cutis laxa have subsequently been found to have abnormal transferring isoelectric focusing and mutations in the ATP6VOA2 gene. ${ }^{139-141}$

\section{Diagnostic testing}

Since the identification of a protein glycosylation defect in 1984 , isoelectric focusing of serum transferrin is the diagnostic screening test of choice. ${ }^{142}$ This method relies on the amount of negative charge on the transferrin related to the number of terminal sialic residues. Type I pattern is characterized by a decrease in the mature tetrasialo-transferrin and an increase of disialo- and asialo-transferrins due to defective assembly of the N-linked glycans in the ER. A type II pattern is characterized by increases of the trisialo- and monosialotransferrin due to a defect in processing. ${ }^{143}$ Since that time, other testing modalities have been employed to recognize deficient glycosylation of serum transferring including capillary electrophoresis, ${ }^{144}$ high-pressure liquid chromatography $\left(\mathrm{HPLC}^{145}\right)$. The utilization of multiple mass spectrometry methods has expanded the diagnostics and characterization of glycosylation. This allows both determination of the number of glycosylated sites and the variety of N-linked glycan structures. These methods include electrospray mass spectrometry (ESI-MS ${ }^{146}$ ), liquid chromatography (LC) coupled with ESI-MS, ${ }^{147,148}$ and matrix assisted laser desorption ionization (MALDI)-MS. ${ }^{149,150}$ 
Isoelectric focusing of serum apolipoprotein C-III (APOC3) can be done to investigate abnormal mucin type O-glycosylation, ${ }^{151}$ which can be seen in some of the combined N- and O-linked glycosylation defects. Immunohistochemistry using antibodies to the glycosylated epitope of alpha-dystroglycan (IIH-6 or VIA4-1) can be utilized to evaluate for dystroglycanopathies..$^{97,152-154}$

Once a defect is suspected, confirmation of the particular gene involved requires molecular analysis. Emerging nextgeneration sequencing of whole exome and whole genome sequencing will aid in identifying defects. ${ }^{155}$

\section{Management/therapy}

Infants and children with all types of protein hypoglycosylation defects require a multidisciplinary approach. Treatment should be tailored to the individual's complications. Nutrition support providing maximal caloric intake and/or nasogastric or gastrostomy tube feedings may be necessary. A comprehensive multisystemic evaluation should be done and support given as necessary. Therapeutic adjuncts including physical, occupational and speech therapy should be utilized to aid in the medical management. Antiepileptic therapy should be used for seizures.

PMI-CDG (CDG-Ib), which is characterized by hepaticintestinal disease, is the only type of hypoglycosylation defect where a specific therapy exists. Mannose given as 1 gram per kg body weight per day divided into five oral doses normalized hypoproteinemia and coagulation defects and rapidly improves the hypoglycemia and protein-losing enteropathy. ${ }^{156-158}$ In some patients with PMI-CDG (CDG-Ib), heparin therapy can be an alternative to mannose in the treatment of the enteropathy. ${ }^{15}$

There has been a report of correction of the infections and improved neutrophil count with fucose treatment in a patient with SLC35C1-CDG (CDG-IIc) ${ }^{159}$ a defect in the fucose transporter.

For the dystroglycanopathies, overexpression of LARGE ${ }^{160}$ and galgt 2 has been shown to improve glycosylation of alpha-dystroglycan in cells from patients and animal models of muscular dystrophy. ${ }^{161}$ In addition, patients with dystroglycanopathies have responded to oral steroids with improvement of muscle function. ${ }^{108}$

\section{Conclusion}

The field of glycosylation defects has been rapidly expanding due to improved clinical awareness and biochemical diagnostic techniques. The clinical spectrum for defects in both $\mathrm{N}$ - and O-linked glycosylation is extremely broad, challenging clinicians to screen for these defects in a variety of settings and disciplines. With some 500 genes involved in the synthesis and function of glycoproteins, it is likely that many more defects have yet to be identified.

\section{Disclosure}

Dr Sparks receives grant funding from NIH/NIAMS and MDA.

\section{References}

1. Varki A, Marth J. Oligosaccharides in vertebrate development. Dev. Biol. 1995;6:127-138.

2. Marquardt T, Denecke J. Congenital disorders of glycosylation: review of their molecular bases, clinical presentations and specific therapies. Eur J Pediatr. Jun 2003;162(6):359-379.

3. Van den Steen P, Rudd PM, Dwek RA, Opdenakker G. Concepts and principles of O-linked glycosylation. Crit Rev Biochem Mol Biol. 1998;33(3):151-208.

4. Chai W, Yuen CT, Kogelberg H, et al. High prevalence of 2-monoand 2,6-di-substituted manol-terminating sequences among O-glycans released from brain glycopeptides by reductive alkaline hydrolysis. Eur J Biochem. Aug 1999;263(3):879-888.

5. Jaeken J, Matthijs G. Congenital disorders of glycosylation. Annu Rev Genomics Hum Genet. 2001;2:129-151.

6. Jaeken J, Matthijs G. From glycosylation to glycosylation diseases. Biochim Biophys Acta. Sep 2009;1792(9):823.

7. Grunewald S, Matthijs G, Jaeken J. Congenital disorders of glycosylation: a review. Pediatr Res. Nov 2002;52(5):618-624.

8. Krasnewich D, O'Brien K, Sparks S. Clinical features in adults with congenital disorders of glycosylation type Ia (CDG-Ia). Am $J$ Med Genet C Semin Med Genet. August 15, 2007;145C(3): 302-306.

9. van de Kamp JM, Lefeber DJ, Ruijter GJ, et al. Congenital disorder of glycosylation type Ia presenting with hydrops fetalis. J Med Genet. Apr 2007;44(4):277-280.

10. Barone R, Sturiale L, Fiumara A, Uziel G, Garozzo D, Jaeken J. Borderline mental development in a congenital disorder of glycosylation (CDG) type Ia patient with multisystemic involvement (intermediate phenotype). J Inherit Metab Dis. Feb 2007;30(1):107.

11. Coman D, McGill J, MacDonald R, et al. Congenital disorder of glycosylation type 1a: three siblings with a mild neurological phenotype. J Clin Neurosci. Jul 2007;14(7):668-672.

12. Matthijs G, Schollen E, Bjursell C, et al. Mutations in PMM2 that cause congenital disorders of glycosylation, type Ia (CDG-Ia). Hum Mutat. Nov 2000;16(5):386-394.

13. de Koning TJ, Dorland L, van Diggelen OP, et al. A novel disorder of $\mathrm{N}$-glycosylation due to phosphomannose isomerase deficiency. Biochem Biophys Res Commun. April 7, 1998;245(1):38-42.

14. Niehues R, Hasilik M, Alton G, et al. Carbohydrate-deficient glycoprotein syndrome type Ib. Phosphomannose isomerase deficiency and mannose therapy. J Clin Invest. April 1, 1998;101(7): 1414-1420.

15. de Lonlay P, Seta N. The clinical spectrum of phosphomannose isomerase deficiency, with an evaluation of mannose treatment for CDG-Ib. Biochim Biophys Acta. Sep 2009;1792(9):841-843.

16. Korner C, Knauer R, Holzbach U, Hanefeld F, Lehle L, von Figura K. Carbohydrate-deficient glycoprotein syndrome type $\mathrm{V}$ : deficiency of dolichyl-P-Glc:Man9GlcNAc2-PP-dolichyl glucosyltransferase. Proc Natl Acad Sci U S A. October 27, 1998;95(22):13200-13205.

17. Grunewald $S$, Imbach $T$, Huijben $K$, et al. Clinical and biochemical characteristics of congenital disorder of glycosylation type Ic, the first recognized endoplasmic reticulum defect in N-glycan synthesis. Ann Neurol. Jun 2000;47(6):776-781. 
18. Hanefeld F, Korner C, Holzbach-Eberle U, von Figura K. Congenital disorder of glycosylation-Ic: case report and genetic defect. Neuropediatrics. Apr 2000;31(2):60-62.

19. Kahook MY, Mandava N, Bateman JB, Thomas JA. Glycosylation type Ic disorder: idiopathic intracranial hypertension and retinal degeneration. Br J Ophthalmol. Jan 2006;90(1):115-116.

20. Sun L, Eklund EA, Van Hove JL, Freeze HH, Thomas JA. Clinical and molecular characterization of the first adult congenital disorder of glycosylation (CDG) type Ic patient. Am J Med Genet A. August 15, 2005; 137(1):22-26.

21. Miller BS, Freeze HH, Hoffmann GF, Sarafoglou K. Pubertal development in ALG6 deficiency (congenital disorder of glycosylation type Ic). Mol Genet Metab. May 2011;103(1):101-103.

22. Denecke J, Kranz C, von Kleist-Retzow J, et al. Congenital disorder of glycosylation type Id: clinical phenotype, molecular analysis, prenatal diagnosis, and glycosylation of fetal proteins. Pediatr Res Aug 2005;58(2):248-253.

23. Denecke J, Kranz C, Kemming D, Koch HG, Marquardt T. An activated $5^{\prime}$ cryptic splice site in the human ALG3 gene generates a premature termination codon insensitive to nonsense-mediated mRNA decay in a new case of congenital disorder of glycosylation type Id (CDG-Id). Hum Mutat. May 2004;23(5):477-486.

24. Stibler H, Stephani U, Kutsch U. Carbohydrate-deficient glycoprotein syndrome - a fourth subtype. Neuropediatrics. Oct 1995;26(5): 235-237.

25. Korner C, Knauer R, Stephani U, Marquardt T, Lehle L, von Figura K. Carbohydrate deficient glycoprotein syndrome type IV: deficiency of dolichyl-P-Man:Man(5)GlcNAc(2)-PP-dolichyl mannosyltransferase. EMBO J. December 1, 1999;18(23):6816-6822.

26. Imbach T, Schenk B, Schollen E, et al. Deficiency of dolichol-phosphate-mannose synthase-1 causes congenital disorder of glycosylation type Ie. J Clin Invest. Jan 2000;105(2):233-239.

27. Kim S, Westphal V, Srikrishna G, et al. Dolichol phosphate mannose synthase (DPM1) mutations define congenital disorder of glycosylation Ie (CDG-Ie). J Clin Invest. Jan 2000;105(2):191-198.

28. Orlean P. Congenital disorders of glycosylation caused by defects in mannose addition during $\mathrm{N}$-linked oligosaccharide assembly. $J$ Clin Invest. Jan 2000;105(2):131-132.

29. Garcia-Silva MT, Matthijs G, Schollen E, et al. Congenital disorder of glycosylation (CDG) type Ie. A new patient. J Inherit Metab Dis. 2004;27(5):591-600.

30. Dancourt J, Vuillaumier-Barrot S, de Baulny HO, et al. A new intronic mutation in the DPM1 gene is associated with a milder form of CDG Ie in two French siblings. Pediatr Res. June 2006;59(6): 835-839.

31. Kranz C, Denecke J, Lehrman MA, et al. A mutation in the human MPDU1 gene causes congenital disorder of glycosylation type If (CDG-If). J Clin Invest. Dec 2001;108(11):1613-1619.

32. Schenk B, Imbach T, Frank CG, et al. MPDU1 mutations underlie a novel human congenital disorder of glycosylation, designated type If. J Clin Invest. Dec 2001;108(11):1687-1695.

33. Chantret I, Dupre T, Delenda C, et al. Congenital disorders of glycosylation type Ig is defined by a deficiency in dolichylP-mannose:Man7GlcNAc2-PP-dolichyl mannosyltransferase. $J$ Biol Chem. July 12, 2002;277(28):25815-25822.

34. Grubenmann CE, Frank CG, Kjaergaard S, Berger EG, Aebi M, Hennet T. ALG12 mannosyltransferase defect in congenital disorder of glycosylation type lg. Hum Mol Genet. September 15, 2002;11(19): 2331-2339.

35. Thiel C, Schwarz M, Hasilik M, et al. Deficiency of dolichyl-PMan:Man7GlcNAc2-PP-dolichyl mannosyltransferase causes congenital disorder of glycosylation type Ig. Biochem J. October 1, 2002;367(Pt 1): 195-201.

36. Zdebska E, Bader-Meunier B, Schischmanoff PO, et al. Abnormal glycosylation of red cell membrane band 3 in the congenital disorder of glycosylation Ig. Pediatr Res. Aug 2003;54(2): 224-229.
37. Di Rocco M, Hennet T, Grubenmann CE, et al. Congenital disorder of glycosylation (CDG) Ig: report on a patient and review of the literature. J Inherit Metab Dis. 2005;28(6):1162-1164.

38. Eklund EA, Newell JW, Sun L, et al. Molecular and clinical description of the first US patients with congenital disorder of glycosylation Ig. Mol Genet Metab. Jan 2005;84(1):25-31.

39. Kranz C, Basinger AA, Gucsavas-Calikoglu M, et al. Expanding spectrum of congenital disorder of glycosylation Ig (CDG-Ig): sibs with a unique skeletal dysplasia, hypogammaglobulinemia, cardiomyopathy, genital malformations, and early lethality. Am J Med Genet A. June 15 , 2007;143A(12):1371-1378.

40. Chantret I, Dancourt J, Dupre T, et al. A deficiency in dolichyl-Pglucose:Glc1Man9GlcNAc2-PP-dolichyl alpha3-glucosyltransferase defines a new subtype of congenital disorders of glycosylation. $J$ Biol Chem. March 14, 2003;278(11):9962-9971.

41. Schollen E, Frank CG, Keldermans L, et al. Clinical and molecular features of three patients with congenital disorders of glycosylation type Ih (CDG-Ih) (ALG8 deficiency). J Med Genet. Jul 2004;41(7):550-556.

42. Eklund EA, Sun L, Westphal V, Northrop JL, Freeze HH, Scaglia F. Congenital disorder of glycosylation (CDG)-Ih patient with a severe hepato-intestinal phenotype and evolving central nervous system pathology. J Pediatr. Dec 2005;147(6):847-850.

43. Vesela K, Honzik T, Hansikova H, et al. A new case of ALG8 deficiency (CDG Ih). J Inherit Metab Dis. August 18, 2009.

44. Stolting T, Omran H, Erlekotte A, Denecke J, Reunert J, Marquardt T. Novel ALG8 mutations expand the clinical spectrum of congenital disorder of glycosylation type Ih. Mol Genet Metab. Nov 2009;98(3):305-309.

45. Thiel C, Schwarz M, Peng J, et al. A new type of congenital disorders of glycosylation (CDG-Ii) provides new insights into the early steps of dolichol-linked oligosaccharide biosynthesis. J Biol Chem. June 20, 2003;278(25):22498-22505.

46. Wu X, Rush JS, Karaoglu D, et al. Deficiency of UDP-GlcNAc:Dolichol Phosphate N-Acetylglucosamine-1 Phosphate Transferase (DPAGT1) causes a novel congenital disorder of Glycosylation Type Ij. Hum Mutat. Aug 2003;22(2):144-150.

47. Grubenmann CE, Frank CG, Hulsmeier AJ, et al. Deficiency of the first mannosylation step in the N-glycosylation pathway causes congenital disorder of glycosylation type Ik. Hum Mol Genet. March 1, 2004;13(5):535-542.

48. Kranz C, Denecke J, Lehle L, et al. Congenital disorder of glycosylation type Ik (CDG-Ik): a defect of mannosyltransferase I. Am J Hum Genet. Mar 2004;74(3):545-551.

49. Schwarz M, Thiel C, Lubbehusen J, et al. Deficiency of GDPMan:GlcNAc2-PP-dolichol mannosyltransferase causes congenital disorder of glycosylation type Ik. Am J Hum Genet. Mar 2004;74(3):472-481.

50. Dupre T, Vuillaumier-Barrot S, Chantret I, et al. Guanosine diphosphatemannose:GlcNAc2-PP-dolichol mannosyltransferase deficiency (congenital disorders of glycosylation type Ik): five new patients and seven novel mutations. J Med Genet. Nov 2010;47(11):729-735.

51. Frank CG, Grubenmann CE, Eyaid W, Berger EG, Aebi M, Hennet T. Identification and functional analysis of a defect in the human ALG9 gene: definition of congenital disorder of glycosylation type IL. Am J Hum Genet. Jul 2004;75(1):146-150.

52. Weinstein M, Schollen E, Matthijs G, et al. CDG-IL: an infant with a novel mutation in the ALG9 gene and additional phenotypic features. Am J Med Genet A. July 15, 2005;136(2):194-197.

53. Vleugels W, Keldermans L, Jaeken J, et al. Quality control of glycoproteins bearing truncated glycans in an ALG9-defective (CDG-IL) patient. Glycobiology. Aug 2009;19(8):910-917.

54. Kranz C, Jungeblut C, Denecke J, et al. A defect in dolichol phosphate biosynthesis causes a new inherited disorder with death in early infancy. Am J Hum Genet. Mar 2007;80(3):433-440.

55. Imtiaz F, Worthington V, Champion $M$, et al. Genotypes and phenotypes of patients in the UK with carbohydrate-deficient glycoprotein syndrome type 1. J Inherit Metab Dis. Mar 2000;23(2):162-174. 
56. Haeuptle MA, Pujol FM, Neupert C, et al. Human RFT1 deficiency leads to a disorder of N-linked glycosylation. Am J Hum Genet. Mar 2008; 82(3):600-606.

57. Clayton PT, Grunewald S. Comprehensive description of the phenotype of the first case of congenital disorder of glycosylation due to RFT1 deficiency (CDG In). J Inherit Metab Dis. Epub 2009; March 11.

58. Jaeken J, Vleugels W, Regal L, et al. RFT1-CDG: Deafness as a novel feature of congenital disorders of glycosylation. J Inherit Metab Dis. Epub 2009; October 24.

59. Vleugels W, Haeuptle MA, Ng BG, et al. RFT1 deficiency in three novel CDG patients. Hum Mutat. Oct 2009;30(10):1428-1434.

60. Rind N, Schmeiser V, Thiel C, et al. A severe human metabolic disease caused by deficiency of the endoplasmatic mannosyltransferase hALG11 leads to congenital disorder of glycosylation-Ip. Hum Mol Genet. April 15, 2010;19(8):1413-1424.

61. Molinari F, Foulquier F, Tarpey PS, et al. Oligosaccharyltransferasesubunit mutations in nonsyndromic mental retardation. Am J Hum Genet. May 2008;82(5):1150-1157.

62. Garshasbi M, Hadavi V, Habibi H, et al. A defect in the TUSC3 gene is associated with autosomal recessive mental retardation. Am J Hum Genet. May 2008;82(5):1158-1164.

63. Cantagrel V, Lefeber DJ, Ng BG, et al. SRD5A3 is required for converting polyprenol to dolichol and is mutated in a congenital glycosylation disorder. Cell. July 23, 2010;142(2):203-217.

64. Morava E, Wevers RA, Cantagrel V, et al. A novel cerebello-ocular syndrome with abnormal glycosylation due to abnormalities in dolichol metabolism. Brain. Nov 2010;133(11):3210-3220.

65. Kahrizi K, Hu CH, Garshasbi M, et al. Next generation sequencing in a family with autosomal recessive Kahrizi syndrome (OMIM 612713) reveals a homozygous frameshift mutation in SRD5A3. Eur J Hum Genet. Jan 2011;19(1):115-117.

66. Van Geet C, Jaeken J, Freson K, et al. Congenital disorders of glycosylation type Ia and IIa are associated with different primary haemostatic complications. J Inherit Metab Dis. Aug 2001;24(4):477-492.

67. de Cock P, Jaeken J. MGAT2 deficiency (CDG-IIa): the Life of J. Biochim Biophys Acta. Sep 2009;1792(9):844-846.

68. De Praeter CM, Gerwig GJ, Bause E, et al. A novel disorder caused by defective biosynthesis of $\mathrm{N}$-linked oligosaccharides due to glucosidase I deficiency. Am J Hum Genet. Jun 2000;66(6):1744-1756.

69. Peters V, Penzien JM, Reiter G, et al. Congenital disorder of glycosylation IId (CDG-IId) - a new entity: clinical presentation with Dandy-Walker malformation and myopathy. Neuropediatrics. Feb 2002;33(1):27-32.

70. Guillard M, Morava E, de Ruijter J, et al. B4GALT1-congenital disorders of glycosylation presents as a non-neurologic glycosylation disorder with hepatointestinal involvement. $J$ Pediatr. Dec 2011;159(6):1041-1043 e1042.

71. Martinez-Duncker I, Dupre T, Piller V, et al. Genetic complementation reveals a novel human congenital disorder of glycosylation of type II, due to inactivation of the Golgi CMP-sialic acid transporter. Blood. April 1, 2005;105(7):2671-2676.

72. Muntoni F, Brockington M, Torelli S, Brown SC. Defective glycosylation in congenital muscular dystrophies. Curr Opin Neurol. Apr 2004;17(2):205-209.

73. Vajsar J, Schachter H. Walker-Warburg syndrome. Orphanet J Rare Dis. 2006;1:29.

74. Fukuyama Y, Osawa M, Suzuki H. Congenital progressive muscular dystrophy of the Fukuyama type - clinical, genetic and pathological considerations. Brain Dev. 1981;3(1):1-29.

75. Longman $\mathrm{C}$, Brockington $\mathrm{M}$, Torelli $\mathrm{S}$, et al. Mutations in the human LARGE gene cause MDC1D, a novel form of congenital muscular dystrophy with severe mental retardation and abnormal glycosylation of alphadystroglycan. Hum Mol Genet. November 1, 2003;12(21):2853-2861.

76. Clarke NF, Maugenre S, Vandebrouck A, et al. Congenital muscular dystrophy type 1D (MDC1D) due to a large intragenic insertion/deletion, involving intron 10 of the LARGE gene. Eur J Hum Genet. Apr 2011; 19(4):452-457.
77. MacLeod H, Pytel P, Wollmann R, et al. A novel FKRP mutation in congenital muscular dystrophy disrupts the dystrophin glycoprotein complex. Neuromuscul Disord. Apr 2007;17(4):285-289.

78. Mercuri E, Topaloglu H, Brockington M, et al. Spectrum of brain changes in patients with congenital muscular dystrophy and FKRP gene mutations. Arch Neurol. Feb 2006;63(2):251-257.

79. Louhichi N, Triki C, Quijano-Roy S, et al. New FKRP mutations causing congenital muscular dystrophy associated with mental retardation and central nervous system abnormalities. Identification of a founder mutation in Tunisian families. Neurogenetics. Feb 2004;5(1): $27-34$.

80. van Reeuwijk J, Grewal PK, Salih MA, et al. Intragenic deletion in the LARGE gene causes Walker-Warburg syndrome. Hum Genet. Jul 2007;121(6):685-690.

81. Van Reeuwijk J, Olderode-Berends MJ, Van den Elzen C, et al. A homozygous FKRP start codon mutation is associated with WalkerWarburg syndrome, the severe end of the clinical spectrum. Clin Genet. Sep 2010;78(3):275-281.

82. Willer T, Valero MC, Tanner W, Cruces J, Strahl S. O-mannosyl glycans: from yeast to novel associations with human disease. Curr Opin Struct Biol. Oct 2003;13(5):621-630.

83. Beltran-Valero de Bernabe D, Currier S, Steinbrecher A, et al. Mutations in the O-mannosyltransferase gene POMT1 give rise to the severe neuronal migration disorder Walker-Warburg syndrome. Am J Hum Genet. Nov 2002;71(5):1033-1043.

84. van Reeuwijk J, Maugenre S, van den Elzen C, et al. The expanding phenotype of POMT1 mutations: from Walker-Warburg syndrome to congenital muscular dystrophy, microcephaly, and mental retardation. Hum Mutat. May 2006;27(5):453-459.

85. Balci B, Uyanik G, Dincer P, et al. An autosomal recessive limb girdle muscular dystrophy (LGMD2) with mild mental retardation is allelic to Walker-Warburg syndrome (WWS) caused by a mutation in the POMT1 gene. Neuromuscul Disord. Apr 2005;15(4):271-275.

86. Lommel M, Cirak S, Willer T, et al. Correlation of enzyme activity and clinical phenotype in POMT1-associated dystroglycanopathies. Neurology. January 12, 2010;74(2):157-164.

87. Manya H, Chiba A, Yoshida A, et al. Demonstration of mammalian protein O-mannosyltransferase activity: coexpression of POMT1 and POMT2 required for enzymatic activity. Proc Natl Acad Sci U S A. January 13, 2004;101(2):500-505.

88. Akasaka-Manya K, Manya H, Nakajima A, Kawakita M, Endo T. Physical and functional association of human protein O-mannosyltransferases 1 and 2. J Biol Chem. July 14, 2006;281(28):19339-19345.

89. van Reeuwijk J, Janssen M, van den Elzen C, et al. POMT2 mutations cause alpha-dystroglycan hypoglycosylation and Walker-Warburg syndrome. J Med Genet. Dec 2005;42(12):907-912.

90. Mercuri E, D'Amico A, Tessa A, et al. POMT2 mutation in a patient with 'MEB-like' phenotype. Neuromuscul Disord. Jul 2006;16(7):446-448.

91. Yanagisawa A, Bouchet C, Van den Bergh PY, et al. New POMT2 mutations causing congenital muscular dystrophy: identification of a founder mutation. Neurology. September 18, 2007;69(12):1254-1260.

92. Biancheri R, Falace A, Tessa A, et al. POMT2 gene mutation in limbgirdle muscular dystrophy with inflammatory changes. Biochem Biophys Res Commun. November 30, 2007;363(4):1033-1037.

93. Cormand B, Avela K, Pihko H, et al. Assignment of the muscle-eyebrain disease gene to 1p32-p34 by linkage analysis and homozygosity mapping. Am J Hum Genet. Jan 1999;64(1):126-135.

94. Yoshida A, Kobayashi K, Manya H, et al. Muscular dystrophy and neuronal migration disorder caused by mutations in a glycosyltransferase, POMGnT1. Dev Cell. Nov 2001;1(5):717-724.

95. Teber S, Sezer T, Kafali M, et al. Severe muscle-eye-brain disease is associated with a homozygous mutation in the POMGnT1 gene. Eur $J$ Paediatr Neurol. Mar 2008;12(2):133-136.

96. Bouchet C, Gonzales M, Vuillaumier-Barrot S, et al. Molecular heterogeneity in fetal forms of type II lissencephaly. Hum Mutat. Oct 2007;28(10):1020-1027. 
97. Godfrey C, Clement E, Mein R, et al. Refining genotype phenotype correlations in muscular dystrophies with defective glycosylation of dystroglycan. Brain. Oct 2007;130(Pt 10):2725-2735.

98. Mercuri E, Messina S, Bruno C, et al. Congenital muscular dystrophies with defective glycosylation of dystroglycan: a population study. Neurology. May 26, 2009;72(21):1802-1809.

99. Clement EM, Godfrey C, Tan J, et al. Mild POMGnT1 mutations underlie a novel limb-girdle muscular dystrophy variant. Arch Neurol. Jan 2008;65(1):137-141.

100. Toda T, Segawa M, Nomura Y, et al. Localization of a gene for Fukuyama type congenital muscular dystrophy to chromosome 9q31-33. Nat Genet. Nov 1993;5(3):283-286.

101. Toda T, Miyake M, Kobayashi K, et al. Linkage-disequilibrium mapping narrows the Fukuyama-type congenital muscular dystrophy (FCMD) candidate region to $<100 \mathrm{~kb}$. Am J Hum Genet Dec 1996;59(6):1313-1320.

102. Kobayashi K, Nakahori Y, Miyake M, et al. An ancient retrotransposal insertion causes Fukuyama-type congenital muscular dystrophy. Nature. July 23, 1998;394(6691):388-392.

103. Silan F, Yoshioka M, Kobayashi K, et al. A new mutation of the fukutin gene in a non-Japanese patient. Ann Neurol. Mar 2003;53(3): 392-396.

104. de Bernabe DB, van Bokhoven H, van Beusekom E, et al A homozygous nonsense mutation in the fukutin gene causes a WalkerWarburg syndrome phenotype. J Med Genet. Nov 2003;40(11): 845-848.

105. Cotarelo RP, Valero MC, Prados B, et al. Two new patients bearing mutations in the fukutin gene confirm the relevance of this gene in Walker-Warburg syndrome. Clin Genet. Feb 2008;73(2):139-145.

106. Yis U, Uyanik G, Heck PB, et al. Fukutin mutations in non-Japanese patients with congenital muscular dystrophy: less severe mutations predominate in patients with a non-Walker-Warburg phenotype. Neuromuscul Disord. Jan 2011;21(1):20-30.

107. Saredi S, Ruggieri A, Mottarelli E, et al. Fukutin gene mutations in an Italian patient with early onset muscular dystrophy but no central nervous system involvement. Muscle Nerve. Jun 2009;39(6): 845-848.

108. Godfrey C, Escolar D, Brockington M, et al. Fukutin gene mutations in steroid-responsive limb girdle muscular dystrophy. Ann Neurol. Nov 2006;60(5):603-610.

109. Puckett RL, Moore SA, Winder TL, et al. Further evidence of Fukutin mutations as a cause of childhood onset limb-girdle muscular dystrophy without mental retardation. Neuromuscul Disord. May 2009;19(5):352-356.

110. Vuillaumier-Barrot S, Quijano-Roy S, Bouchet-Seraphin C, et al. Four Caucasian patients with mutations in the fukutin gene and variable clinical phenotype. Neuromuscul Disord. Mar 2009;19(3):182-188.

111. Arimura T, Hayashi YK, Murakami T, et al. Mutational analysis of fukutin gene in dilated cardiomyopathy and hypertrophic cardiomyopathy. Circ J. Jan 2009;73(1):158-161.

112. Murakami T, Hayashi YK, Noguchi S, et al. Fukutin gene mutations cause dilated cardiomyopathy with minimal muscle weakness. Ann Neurol. Nov 2006;60(5):597-602.

113. Toda T, Kobayashi K, Takeda S, et al. Fukuyama-type congenital muscular dystrophy (FCMD) and alpha-dystroglycanopathy. Congenit Anom (Kyoto). Jun 2003;43(2):97-104.

114. Xiong H, Kobayashi K, Tachikawa M, et al. Molecular interaction between fukutin and POMGnT1 in the glycosylation pathway of alpha-dystroglycan. Biochem Biophys Res Commun. December 1, 2006;350(4): 935-941.

115. Peyrard M, Seroussi E, Sandberg-Nordqvist AC, et al. The human LARGE gene from 22q12.3-q13.1 is a new, distinct member of the glycosyltransferase gene family. Proc Natl Acad Sci U SA. January 19, 1999; 96(2):598-603.

116. Inamori K, Yoshida-Moriguchi T, Hara Y, Anderson ME, Yu L, Campbell KP. Dystroglycan function requires xylosyl- and glucuronyltransferase activities of LARGE. Science. January 6, 2012; 335(6064):93-96.
117. Eisenberg I, Avidan N, Potikha T, et al. The UDP-N-acetylglucosamine 2-epimerase/ $\mathrm{N}$-acetylmannosamine kinase gene is mutated in recessive hereditary inclusion body myopathy. Nat Genet. Sep 2001; 29(1):83-87.

118. Keppler OT, Hinderlich S, Langner J, Schwartz-Albiez R, Reutter W, Pawlita M. UDP-GlcNAc 2-epimerase: a regulator of cell surface sialylation. Science. May 21, 1999;284(5418):1372-1376.

119. Argov Z, Yarom R. "Rimmed vacuole myopathy" sparing the quadriceps. A unique disorder in Iranian Jews. J Neurol Sci. Apr 1984; 64(1):33-43.

120. Sadeh M, Gadoth N, Hadar H, Ben-David E. Vacuolar myopathy sparing the quadriceps. Brain. Feb 1993;116(Pt 1):217-232.

121. Nonaka I, Sunohara N, Ishiura S, Satoyoshi E. Familial distal myopathy with rimmed vacuole and lamellar (myeloid) body formation. J Neurol Sci. Jul 1981;51(1):141-155.

122. Nishino I, Noguchi S, Murayama K, et al. Distal myopathy with rimmed vacuoles is allelic to hereditary inclusion body myopathy. Neurology. December 10, 2002;59(11):1689-1693.

123. Lesnik Oberstein SA, Kriek M, White SJ, et al. Peters Plus syndrome is caused by mutations in B3GALTL, a putative glycosyltransferase. Am J Hum Genet. Sep 2006;79(3):562-566.

124. Specktor P, Cooper JG, Indelman M, Sprecher E. Hyperphosphatemic familial tumoral calcinosis caused by a mutation in GALNT3 in a European kindred. J Hum Genet. 2006;51(5):487-490.

125. Lefeber DJ, Schonberger J, Morava E, et al. Deficiency of Dol-P-Man synthase subunit DPM3 bridges the congenital disorders of glycosylation with the dystroglycanopathies. Am J Hum Genet. July 2009; 85(1):76-86.

126. Etzioni A, Sturla L, Antonellis A, et al. Leukocyte adhesion deficiency (LAD) type II/carbohydrate deficient glycoprotein (CDG) IIc founder effect and genotype/phenotype correlation. AmJMed Genet. June 15, 2002; 110(2):131-135.

127. Wu X, Steet RA, Bohorov O, et al. Mutation of the COG complex subunit gene COG7 causes a lethal congenital disorder. Nat Med. May 2004; 10(5):518-523.

128. Spaapen LJ, Bakker JA, van der Meer SB, et al. Clinical and biochemical presentation of siblings with COG-7 deficiency, a lethal multiple O- and N-glycosylation disorder. J Inherit Metab Dis. 2005; 28(5):707-714

129. Morava E, Zeevaert R, Korsch E, et al. A common mutation in the COG7 gene with a consistent phenotype including microcephaly, adducted thumbs, growth retardation, VSD and episodes of hyperthermia. Eur J Hum Genet. Jun 2007;15(6):638-645.

130. Ng BG, Kranz C, Hagebeuk EE, et al. Molecular and clinical characterization of a Moroccan Cog7 deficient patient. Mol Genet Metab. Jun 2007;91(2):201-204.

131. Foulquier F, Vasile E, Schollen E, et al. Conserved oligomeric Golgi complex subunit 1 deficiency reveals a previously uncharacterized congenital disorder of glycosylation type II. Proc Natl Acad Sci USA. March 7, 2006;103(10):3764-3769.

132. Foulquier F, Ungar D, Reynders E, et al. A new inborn error of glycosylation due to a $\operatorname{Cog} 8$ deficiency reveals a critical role for the Cog1-Cog8 interaction in COG complex formation. Hum Mol Genet. April 1, 2007;16(7):717-730.

133. Kranz C, Ng BG, Sun L, et al. COG8 deficiency causes new congenital disorder of glycosylation type IIh. Hum Mol Genet. April 1, 2007; 16(7):731-741.

134. Reynders E, Foulquier F, Leao Teles E, et al. Golgi function and dysfunction in the first COG4-deficient CDG type II patient. Hum Mol Genet. September 1, 2009;18(17):3244-3256.

135. Miura Y, Tay SK, Aw MM, Eklund EA, Freeze HH. Clinical and biochemical characterization of a patient with congenital disorder of glycosylation (CDG) IIx. J Pediatr. Dec 2005;147(6): $851-853$

136. Ng BG, Sharma V, Sun L, et al. Identification of the first COGCDG patient of Indian origin. Mol Genet Metab. Mar 2011; 102(3):364-367. 
137. Paesold-Burda P, Maag C, Troxler H, et al. Deficiency in COG5 causes a moderate form of congenital disorders of glycosylation. Hum Mol Genet. November 15, 2009;18(22):4350-4356.

138. Lubbehusen J, Thiel C, Rind N, et al. Fatal outcome due to deficiency of subunit 6 of the conserved oligomeric Golgi complex leading to a new type of congenital disorders of glycosylation. Hum Mol Genet. September 15, 2010;19(18):3623-3633.

139. Van Maldergem L, Ogur G, Yuksel M. Facial anomalies in congenital cutis laxa with retarded growth and skeletal dysplasia. Am JMed Genet. Feb 1989;32(2):265.

140. Kornak U, Reynders E, Dimopoulou A, et al. Impaired glycosylation and cutis laxa caused by mutations in the vesicular H+-ATPase subunit ATP6V0A2. Nat Genet. Jan 2008;40(1):32-34.

141. Morava E, Wopereis S, Coucke P, et al. Defective protein glycosylation in patients with cutis laxa syndrome. Eur J Hum Genet. Apr 2005; 13(4):414-421.

142. Jaeken J, van Eijk HG, van der Heul C, Corbeel L, Eeckels R, Eggermont E. Sialic acid-deficient serum and cerebrospinal fluid transferrin in a newly recognized genetic syndrome. Clin Chim Acta. December 29, 1984;144(2-3):245-247.

143. Jaeken J. Komrower Lecture. Congenital disorders of glycosylation (CDG): it's all in it! J Inherit Metab Dis. 2003;26(2-3):99-118.

144. Carchon HA, Chevigne R, Falmagne JB, Jaeken J. Diagnosis of congenital disorders of glycosylation by capillary zone electrophoresis of serum transferrin. Clin Chem. Jan 2004;50(1):101-111.

145. Helander A, Eriksson G, Stibler H, Jeppsson JO. Interference of transferrin isoform types with carbohydrate-deficient transferrin quantification in the identification of alcohol abuse. Clin Chem. 2001; 47(7):1225-1233.

146. Wada Y, Nishikawa A, Okamoto N, et al. Structure of serum transferrin in carbohydrate-deficient glycoprotein syndrome. Biochem Biophys Res Commun. December 15, 1992;189(2):832-836.

147. Lacey JM, Bergen HR, Magera MJ, Naylor S, O’Brien JF. Rapid determination of transferrin isoforms by immunoaffinity liquid chromatography and electrospray mass spectrometry. Clin Chem. Mar 2001; 47(3):513-518.

148. Bergen HR, Lacey JM, O’Brien JF, Naylor S. Online single-step analysis of blood proteins: the transferrin story. Anal Biochem. September 1, 2001;296(1):122-129.

149. Wada Y, Gu J, Okamoto N, Inui K. Diagnosis of carbohydratedeficient glycoprotein syndrome by matrix-assisted laser desorption time-of-flight mass spectrometry. Biol Mass Spectrom. Feb 1994; 23(2):108-109.
150. Sturiale L, Barone R, Garozzo D. The impact of mass spectrometry in the diagnosis of congenital disorders of glycosylation. J Inherit Metab Dis. Aug 2011;34(4):891-899.

151. Wopereis S, Morava E, Grunewald S, et al. Patients with unsolved congenital disorders of glycosylation type II can be subdivided in six distinct biochemical groups. Glycobiology. Dec 2005;15(12): 1312-1319.

152. Muntoni F, Valero de Bernabe B, Bittner R, et al. 114th ENMCInternational Workshop on Congenital Muscular Dystrophy (CMD) January 17-19, 2003, Naarden, The Netherlands: (8th Workshop of the International Consortium on CMD; 3rd Workshop of the MYO-CLUSTER project GENRE). Neuromuscul Disord. Sep 2003; 13(7-8):579-588.

153. Muntoni F, Torelli S, Brockington M. Muscular dystrophies due to glycosylation defects. Neurotherapeutics. Oct 2008;5(4):627-632.

154. Hewitt JE. Abnormal glycosylation of dystroglycan in human genetic disease. Biochim Biophys Acta. Sep 2009;1792(9):853-861.

155. Lefeber DJ, Morava E, Jaeken J. How to find and diagnose a CDG due to defective N-glycosylation. J Inherit Metab Dis. Aug 2011; 34(4):849-852.

156. Harms HK, Zimmer KP, Kurnik K, Bertele-Harms RM, Weidinger S, Reiter K. Oral mannose therapy persistently corrects the severe clinical symptoms and biochemical abnormalities of phosphomannose isomerase deficiency. Acta Paediatr. 2002;91(10):1065-1072.

157. Mention K, Lacaille F, Valayannopoulos V, et al. Development of liver disease despite mannose treatment in two patients with CDG-Ib. Mol Genet Metab. Jan 2008;93(1):40-43.

158. de Lonlay P, Cuer M, Vuillaumier-Barrot S, et al. Hyperinsulinemic hypoglycemia as a presenting sign in phosphomannose isomerase deficiency: A new manifestation of carbohydrate-deficient glycoprotein syndrome treatable with mannose. J Pediatr. Sep 1999; 135(3):379-383.

159. Marquardt T, Luhn K, Srikrishna G, Freeze HH, Harms E, Vestweber D. Correction of leukocyte adhesion deficiency type II with oral fucose. Blood. December 15, 1999;94(12):3976-3985.

160. Barresi R, Michele DE, Kanagawa M, et al. LARGE can functionally bypass alpha-dystroglycan glycosylation defects in distinct congenital muscular dystrophies. Nat Med. Jul 2004;10(7):696-703.

161. Martin PT. Congenital muscular dystrophies involving the O-mannose pathway. Curr Mol Med. Jun 2007;7(4):417-425.
The Application of Clinical Genetics

\section{Publish your work in this journal}

The Application of Clinical Genetics is an international, peer-reviewed open access journal that welcomes laboratory and clinical findings in the field of human genetics. Specific topics include: Population genetics; Functional genetics; Natural history of genetic disease; Management of genetic disease; Mechanisms of genetic disease; Counselling and

\section{Dovepress}

ethical issues; Animal models; Pharmacogenetics; Prenatal diagnosis; Dysmorphology. The manuscript management system is completely online and includes a very quick and fair peer-review system, which is all easy to use. Visit http://www.dovepress.com/testimonials.php to read real quotes from published authors. 